\title{
Resource utilization of composite insulator silicone rubber
}

\author{
Yuze Jiang ${ }^{l, *}$, Yong Zhang ${ }^{l}$, Diwen Jiang ${ }^{2}$, Chao Wang ${ }^{l}$, Yimin Chen ${ }^{l}$ \\ ${ }^{1}$ State Grid Shandong Electric Power Research Institute, Jinan, 250003, China \\ ${ }^{2}$ State Grid Jinan Jiyang Power Supply Company, Jinan, 251400, China
}

\begin{abstract}
Composite insulators provide highly effective insulation support, and their application in power transmission lines has seen rapid growth in recent years. However, every year, a large quantity of composite insulators are scrapped on reaching the end of their service life. Due to the exceptional chemical inertness of the silicone rubber in the insulators, they have poor self-degradation characteristics, and end up filling landfills and occupying a lot of space, which inadvertently endangers the ecological environment. So far, there has not been any effective means to recycle composite insulators. This paper analyzes the material composition and characteristics of the silicone rubber in the core and housing of composite insulators, and proposes a resource utilization approach for the application of the pulverized silicone rubber powder from the insulators in the following three applications: use in producing silicone rubber asphalt at a blending ratio of $15 \%-18 \%$ - the corresponding market demand is large enough to meet the disposal needs of scrapped composite insulators; use in modification of waste rubber powder and blending with ethylene propylene diene monomer rubber (EPDM) at a blending ratio of 10\% - as the annual production capacity of EPDM is more than 850,000 tons in China, thousands of tons of waste insulators can be thus disposed; and use as an aggregate for non-slip coatings, which accounts for more than $40 \%$ of the coatings material. Thus, there is tremendous scope for recycling waste insulator.
\end{abstract}

\section{Introduction}

Composite insulators have various advantages, such as simple molding and other production processes, low cost, light weight, etc., and when compared with ceramic and glass insulators, they have various advantages (high mechanical strength, strong hydrophobicity, good pollution flashover resistance and good self-cleaning properties) that solve the pollution flashover problem of the external insulation for transmission lines in polluted areas, and also reduce the workload of transportation and maintenance for pollution cleaning and zero value detection. Therefore, in recent years, composite insulators have been widely used in AC and DC transmission lines of various voltage levels ${ }^{[1-7]}$. According to statistics given in Chao et al. (2015), the number of composite insulators in operation has exceeded $45 \%$ of the total number of insulators on the transmission lines of the State Grid and the Southern Power Grid; in some provinces, the number is more than $70 \%{ }^{[1]}$. These statistics indicate that composite insulators are the most commonly used type of insulators among all types. As the voltage of transmission lines increases, there is an increased requirement for external insulation, resulting in a proportionate increase in composite insulator utilization. In the $3 \mathrm{AC}$ UHV lines operated in the early days, composite insulators accounted for about $60 \%$; in the 6 DC UHV lines, they are estimated to account for more than $85 \%$ of the insulators used ${ }^{[7]}$. From 1995 onwards, composite insulators have been widely promoted and used; in the same year, there were 100,000 pcs of composite insulators in operation $\left.{ }^{[5}\right]$. Since then the number of composite insulators utilized in China has shown explosive growth; at the end of 2013, more than 6 million pcs of composite insulators were in operation $^{[1]}$. At present, there are more than 180 composite insulator manufacturers of medium scale size and above, and the annual output of several large-scale manufacturers exceeds $300,000 \mathrm{pcs}^{[8]}$, with the annual output of the entire industry estimated to be in millions or even tens of millions of pcs.

The sheds and housing of the composite insulator are made from a special synthetic silicone rubber. Under the combined effects of temperature, pollution, humidity, partial discharge, or high field strength, aging of rubber occurs, manifesting in the form of fracturing, pulverization, reduced hydrophobicity, cracking, etc., during its operation. This is a serious threat to the insulation performance of power transmission and transformation equipment. Experimental studies and operational experience show that the service life of composite insulator silicone rubber materials is generally 15-20 years under normal design, manufacturing, use, and maintenance conditions ${ }^{[1][9-10]}$. It has been 25 years since composite insulators were introduced, and many batches of composite insulators have reached their service life and have been scrapped. In future, more and more insulators will be scrapped each year. At the same time, composite insulators were also scrapped mid-life due to abnormal aging or early failure, caused by design

\footnotetext{
* Corresponding author: jiangyuze@163.com
} 
defects of formula, poor raw materials, manufacturing defects, unreasonable selection, lightning strike, bird damage, pollution flashover, heavy non-soluble deposit density(NSDD), environmental conditions, etc ${ }^{[1][5]}$. Since the number of applications is large, the number of scrapped composite insulators is correspondingly large as well.

Silicone rubber has a main chain of silicon-oxygen bond and has excellent chemical inertness (i.e. high and low temperature resistance, high voltage resistance, ozone aging resistance, radiation resistance, and weather resistance) due to its structural particularity, hence, it finds wide use in various application fields. Due to its exceptional advantages, the disposal of scrapped composite insulators is difficult. As insulators continuously accumulate in landfills, endangering the safety of the ecological environment, there is an urgent need for an appropriate disposal solution for scrapped composite insulators. Resource utilization of scrapped composites has important implications for environmental protection, and for the healthy and sustainable development of the composite insulators industry. However, the comprehensive utilization of scrapped composite insulators has just started and being in the exploratory stage, no clear-cut practical method of treatment has found acceptance as yet.

\section{Composite insulator silicone rubber material and composi-tion}

The silicone rubber material used in composite insulator is high temperature vulcanized (HTV) silicone rubber, the raw materials of which mainly include polymethyl vinyl siloxane (referred to as methyl vinyl silicone rubber or raw silicone rubber), reinforcing filler, constitution controller, colorant, crosslinking agent, vulcanizing agent, modified additive, etc. The reinforcing filler is fumed silica. Commonly used constitution controllers are cyclo-silazane, hexamethyldisilazane, diphenyl silicon glycol, and hydroxy silicone oil with low molar mass, etc. Commonly used crosslinking agents are vinyl polysiloxane and polymethylhydrosiloxane. Ferric oxide is used as a red colorant or used along with carbon for black color. Commonly used vulcanizing agents are: benzoyl peroxide, 2,4-dichlorobenzoyl peroxide, tertbutyl peroxybenzoate, di-tert-butyl peroxide, dicumyl peroxide, 2,5-dimethyl-2,5-di-tert-butyl peroxy-hexane, etc. The modified additive is aluminum hydroxide, which is used to increase the resistance to electric trace level and vertical combustion grade of the insulator ${ }^{[11-14]}$.

The ratio range of each component for silicone rubber to be used in sheds and housing are: 100 parts of methyl vinyl silicone rubber, 30-40 parts of reinforcing filler (silica), 90-120 parts of aluminum hydroxide, 1.52.5 parts of vulcanizing agent, 3-4 parts of crosslinking agent, 4-6 parts of constitution controller ${ }^{[13-14]}$. It can be seen from the ratio of silicone rubber that organic components (i.e. raw rubber, crosslinking agent, and constitution controller) in silicone rubber account for about $40 \%$, while inorganic components (i.e. aluminum hydroxide, silica and colorant) account for about $60 \%$.
The ratio range of each component for silicone rubber to be used in sheds and housing are: 100 parts of methyl vinyl silicone rubber, 30-40 parts of reinforcing filler (silica), 90-120 parts of aluminum hydroxide, 1.52.5 parts of vulcanizing agent, 3-4 parts of crosslinking agent, 4-6 parts of constitution controller ${ }^{[13-14]}$. It can be seen from the ratio of silicone rubber that organic components (i.e. raw rubber, crosslinking agent, and constitution controller) in silicone rubber account for about $40 \%$, while inorganic components (i.e. aluminum hydroxide, silica and colorant) account for about $60 \%$.

\section{Analysis of suitable ways to use insulator silicone rubber}

The silicone rubber material used in the composite insulator is designed and developed to meet the special functional requirements of the suspended external insulation protection of the transmission lines. Its main material (methyl vinyl silicone rubber) is the same as that used in other silicone rubber products except for the additives, which are specifically selected, and the proportions thereof are adjusted to achieve the external insulation function required.

The inorganic components of the composite insulator do not change during operation, and the aging of the insulator is in fact the aging, pyrolysis and loss of the organic components of the silicone rubber in the sheds and housing. Therefore, the content of organic components in scrapped composite insulators are found to be reduced to below $40 \%$, while the proportion of inorganic components are found to have increased to above $60 \%$.

There are two main methods for the processing of waste rubber in other industries: one is the pulverization method, in which the resulting waste silicone rubber powder is used as additives for organic resins, thermoplastic elastomers, building materials, cosmetics, inks, coatings, and flame retardant materials; the other is the pyrolysis method, by which the waste silicone rubber is converted to rubber materials that can be used for further processing and vulcanization, or depolymerized to a cyclic siloxane intermediate (DMC) or even a siloxane monomer. Despite the high value of the products recovered in this process, the pyrolysis method is complex, has high costs, and high energy consumption. The recyclable part of composite insulator waste silicone rubber disposed by consuming huge energy only accounts for less than $40 \%$ of the total processing load, thereby requiring recycling or disposal of the remaining part, which obviously increases the recycling cost greatly; hence this is not an economically appropriate approach. Therefore, the pulverization method should be considered preferentially for the comprehensive utilization of scrapped composite insulators to process silicone rubber powder used in the following fields:

\subsection{Silicone rubber asphalt}


Rubber asphalt is a composite cementing material formed by the full swelling reaction of rubber powder and asphalt at high temperature. Data from a large number of applications shows that asphalt concrete modified with rubber powder has low temperature sensitivity, good resistance to rutting deformation and cold cracking, and can increase the service life of the pavement. It also makes the pavement smooth and seamless, increases driving comfort, has low vibration and low noise, good water damage resistance, wear resistance and wet skid resistance, and improves the driving safety factor. Moreover, it leads to a short construction period and simple maintenance. Furthermore, the relatively cheaper price of waste rubber powder can reduce the cost of road construction ${ }^{[15-16]}$. Statistically, the average service life of rubber asphalt pavement is longer than that of common asphalt pavement by $2-5$ years or $30-80 \%{ }^{[16]}$. Due to its excellent engineering properties, rubber asphalt has been widely recognized and used in road projects all over the world $^{[30]}$.

At present, rubber asphalt is applied using a wet process, and the addition amount of asphalt is $15-18.5 \%$ ${ }^{[17-19]}$. Statistically, as many as 4000 waste tires are consumed per kilometer of rubber asphalt pavement ${ }^{[15][20]}$. So the use of rubber asphalt in road paving has created a huge demand for rubber powder. In China, there is a serious shortage of waste tire supply (the usual source of waste rubber powder) for rubber asphalt production due to its large territory and the substantial number of road construction projects underway. Accordingly, other modified asphalts have had to be used instead ${ }^{[21]}$.

The mechanism of asphalt modification by rubber powder is as follows: 1) After addition, the rubber powder adsorbs the aromatic hydrocarbons and saturated hydrocarbons from the asphalt components, due to which the asphalt compounds come into close proximity with the polymer chain of rubber. The rubber powder is randomly distributed in the asphalt matrix as particles or filaments. The rubber powders form a certain networked structure due to their adhering to each other, and the asphalt is changed from a homogenous body into a twophase continuous structure blended system; 2) As asphalt contains both polar and non-polar compounds, as well as organic functional groups (hydroxyl groups, aliphatic groups, etc.), chemical blending occurs between the asphalt and rubber powder initiating a chemical reaction ${ }^{[19][22-26]}$. In the initial stage of the reaction, the swelling effect of the rubber powder is dominant, and the rubber powder forms a loose flocculent structure; at the same time, light oil is absorbed by the rubber powder, and the viscosity of the asphalt increases to gradually cause the mutual dissolution of the two phases. After the swelling reaches a certain stage, the light components in the asphalt enter into the gaps of the rubber chain to accelerate the development of desulfurization and degradation, then partially dissolve the rubber powder and distribute more uniformly, which results in a decrease in viscosity as compared to the previous stage; finally, the rubber powder is connected via the gel film to form a semi-solid continuous phase system with high viscosity, which significantly increases the viscosity of the rubber asphalt. The increased viscosity leads to an increase in the thickness of the asphalt film coated on the aggregates, thereby improving the resistance of the asphalt mixture to oxidation, temperature changes, and fatigue cracking.

The reason for the improvement of high-temperature stability is that rubber asphalt has a higher value for high-temperature viscosity than pure asphalt. The rubber powder swells at high temperature, which increases the internal friction angle of the mixture, so the resistance to high-temperature cracking is improved. The reason for the improvement of resistance to low-temperature cracking is that mixing rubber with asphalt reduces the stiffness modulus of the mixture at low temperature and improves the ultimate tensile strength of the rubber asphalt mixture. The reason for the improvement of resistance to water damage is that the rubber asphalt forms a suitable oil film protective layer on the aggregates' surface, which prevents easy leakage and flooding of oil, while the protection provided by the oil film improves the resistance to water damage of the road, and similarly improve low-temperature performance. As the elastic modulus of rubber asphalt mixture decreases, the bending and tensile stress of the material also decreases, so that under the action of dynamic loading, the dynamic response capacity of the mixture improves, thereby extending the fatigue life of the road surface ${ }^{[26]}$.

Based on the aforementioned rubber modification mechanism, it can be seen that after the addition of insulator silicone rubber powder to the asphalt matrix, the reaction mentioned above to improve the asphalt performance can occur completely, which in turn modifies the asphalt, making it feasible to use the insulator silica gel powder to produce rubber asphalt. Shen et $a l^{[27]}$. carried out a study on the preparation and performance of silicone rubber powder modified asphalt, wherein silicone rubber powder was mixed into No.70 and No.90 matrix asphalt to prepare modified asphalt. Results: under the condition of 180 mesh silicone rubber powder, $20 \%$ addition, and $180^{\circ} \mathrm{C}$ stirring temperature, the high and low-temperature performance of the rubber asphalt produced were optimal. The softening point of modified asphalt increased by $14.66 \%$ (No.70) and about $12.84 \%$ (No.90), which improved the high-temperature performance of the modified asphalt. In addition, the penetration of modified asphalt reduced by $14.99 \%$ (No.70) and 33.68\% (No.90), indicating that the modified asphalt was more pressure-resistant. The ductility $\left(5^{\circ} \mathrm{C}\right)$ of modified asphalt increased by $33 \mathrm{~mm}$ (No.70) and $51 \mathrm{~mm}$ (No.90), which improved the lowtemperature performance of the modified asphalt. The experimental results show that it is feasible to use silicone rubber for asphalt modification. Furthermore, there is a supply gap of rubber powder in the market, so the engineering application study on scrapped composite insulators for asphalt modification should be fully developed as soon as possible.

\subsection{Modification of waste rubber powder and blending with EPDM}


Ethylene propylene diene monomer (EPDM) is a copolymer of ethylene, propylene, and a small amount of non-conjugated diene. As its main chain is composed of chemically stable saturated hydrocarbons, it has excellent aging resistance (ozone resistance, oxidative resistance, abrasive resistance, and weather resistance), water resistance, chemical resistance, good gas impermeability, and excellent electrical insulation property. It is widely used in the manufacture of automobile parts, waterproof materials for construction, wire and cable sheaths, heat resistant hoses, heat resistant conveyor belts, tapes, automobile seals, door and window sealing strips, anticorrosive linings, household appliance accessories, etc.

Blending silicone rubber with EPDM further improves the heat resistance and elasticity, cold resistance, and processability of the rubber. This process acquires the excellent performance of both of the constituents by improving the insulative properties, specifically heat insulation. As early as in the 1990s, silicone rubber/EPDM blended products, such as the JSR JENIXE series (from JSR) and the TEO series (from Toshiba Silicone) have been available in the international market ${ }^{[28-29]}$.

Dou et al. conducted a study on the blending of methyl vinyl silicone rubber (MVQ) and EPDM, and concluded that the tear strength and elongation of blends at break-even exceed the EPDM rubber compound at a blending ratio (MVQ/EPDM) range of 20/80 - 40/60 [30]. After addition, silica has a strong reinforcing effect. Both methyl vinyl silicone rubber and silica are the main components in insulator silicone rubber, which indicates that it is feasible to use waste insulators for EPDM blending. Zhang et $\mathrm{al}^{[31-32]}$. conducted studies on the blending of modified waste silicone rubber composite insulator powder and EPDM: Considering cost and performance comprehensively, a 90 silicone rubber : 10 EPDM blending ratio for EPDM-activated modified waste silicone rubber was found to be better. After addition of mixed modifiers (modified rubber powder) the tensile strength and elongation at the break point of the blends increased by $16.5 \%$ and $11.2 \%$, respectively. The tear strength reached $34.0 \mathrm{kN} / \mathrm{m}$, and the comprehensive mechanical properties were found to be good. In conclusion, it is feasible to produce EPDM products by blending scrapped waste insulator rubber powder with EPDM at a mixing ratio of about $10 \%$. It can reduce the production cost of blended products and improve its economic benefits by replacing expensive silicon rubber powder raw materials with inexpensive waste insulator silicon rubber powder.

Based on the "2013-2018 China EPDM Market Survey and Development Prospect Consultation Report" issued by ASKCI, the total EPDM manufacturing capacity of more than 10 new ethylene propylene rubber projects in China by domestic and foreign manufacturers is close to 850,000 tons/year. In contrast, the domestic market demand of EPDM is a little more than 320,000 tons/year. Production of EPDM-blended products has great potential for the disposal of waste insulators; the disposed amount of waste insulator silicone rubber through this means can reach tens of thousands of tons annually. This throughput could account for half of the production capacity of composite insulators, keeping in mind that the demand of the composite silicone rubber insulator industry is in the order of 70,000 tons per year.

\subsection{Skid-resistant coatings}

Skid-resistant coating is a kind of functional coating that has increasing application. Skid-resistant coatings are mainly composed of film-forming resin, additives, skidresistant aggregate, solvent, etc. As an important component of skid-resistant coating, skid-resistant aggregates can effectively improve the friction of the pavement and provide reliable friction to reduce the sliding of people and vehicles on the pavement. Skidresistant aggregate materials can be divided into two kinds: synthetic organic materials and inorganic materials. Among the commonly used synthetic organic skid-resistant aggregates are inert polymer materials, such as polyethylene, polypropylene resin particles, rubber particles, polyurethane resin particles, etc ${ }^{[33]}$. Common inorganic skid-resistant aggregates include silica powder, bauxite, quartz sand, glass beads, emery, silicon carbide, crystalline alumina, mica, etc.

Due to its excellent chemical inertness, it is difficult to dispose scrapped composite insulators, but this property meets the requirement of organic aggregates used in skid-resistant coating. At the same time, the main additive used in silicone rubber insulators-aluminum hydroxide, can be used as an inorganic skid-resistant aggregate. Natural rubber, waste tire powder, and polyurethane particles are the most commonly used elastic skid-resistant particles, which can not only increase the skid resistance of the coating ${ }^{[34]}$, but also improve the comfort level during treading and contact of the product surfaces. Therefore, using silicone rubber to produce skid-resistant coatings is feasible. Skid-resistant coatings are more and more widely used in various industries, and skid-resistant aggregates account for 42 $54 \%$ of skid-resistant coatings ${ }^{[35]}$. By using silicone rubber for skid-resistant coatings, it can consume large amounts of scrapped composite insulator silicone rubber sheds. This application has good prospects.

\section{Conclusion}

This study briefly summarizes the application of composite insulators, the present status, and the urgent need for resource utilization of scrapped insulators, and summarizes and analyzes the composition and characteristics of insulator materials. Considering that more than $60 \%$ of materials in composite insulators are inorganic, the study proposes the preferential application of pulverization for scrapped composite insulators. The obtained rubber powder can be used in the following three ways:

1) Used in the production of silicone rubber asphalt at an addition ratio of about $15-18 \%$, which has large market demand and faces a current shortage of waste tire rubber powder supply; 
2) Used in the production of EPDM-blended products at an addition ratio of about $10 \%$. The disposal capacity for scrapped composite insulators by this means will reach tens of thousands of tons per year, which accounts for almost half the production capacity of the insulator industry;

3) Used in skid-resistant coatings. The main organic material of scrapped composite insulators (silicone rubber) meets the requirement of organic aggregates required in skid-resistant coatings and contains an inorganic aggregate component (aluminum hydroxide). Skid-resistant coatings are used more and more widely in various industries, and skid-resistant aggregates account for more than $40 \%$ of the skid-resistant coatings, thereby creating a huge demand for waste insulators.

Today, as the pressure for environmentally-friendly disposal of scrapped composite insulators is increasing sharply, the development of these specific technologies for recycling composite insulators in the manners mentioned above should be carried out as soon as possible for speedy realization of commercial applications.

\section{References}

1. Chao Yafeng, Wang Cheng, Huang Fuyong, Zhou Weihua. Analysis of the operation status of composite insulators for transmission lines in China [J]. High Voltage Apparatus. 2015,51(8):119-124.

2. Liu Yang, Wang Jianguo, Han Fang, et al. Electrical and mechanical properties of composite insulators with different operating years [J]. High Voltage Engineering, 2008, 34(5): 1017-1021.

3. Wang Shaowu. Study on the characteristics of organic external insulation in polluted areas [D]. Beijing: Tsinghua University, 2001: 33-34.

4. Cao Hongwei, Zhang Weijie, Guo Mingjia, etc. Analysis of pollution components and research on cleaning technology for scrapped composite insulators [J]. Applied Chemical Industry, 2017, 46(12) 2403-2407.

5. Liu Zehong. Status quo of composite insulators use and application prospects thereof in UHV transmission lines $[\mathrm{J}]$. Power System Technology, 2006, 30(12):1-7.

6. Wang Jingyi. Discussion on the application of composite insulators in UHV DC projects [J]. Power System and Clean Energy, 2010, 26(5): 15-18.

7. Liang Xidong, Gao Yanfeng, Wang Jiafu, Li Shaohua. The rapid development history of China's silicone rubber composite insulators [J]. High Voltage Engineering, 2016, 42(9): 2888-2896.

8. Wu Guangya, Peng Jiankun, Zhang Rui. Status quo and application prospects of composite insulators market in China [J]. Electrical Equipment, 2005. 6(3): 104-106.

9. Su Zhiyi, Chen Gang, Li Qingfeng, et al. Study on the aging and criterion of sheds and housing of silicone rubber composite insulator [J]. Power System Technology, 2006, 30(12): 53-57.

10. Yan Nianping, Jiafang Wei, Wan Hua, Cheng Zheng, Jia Zhidong. Study progress on the aging status and characterization technology of high-temperature vulcanized silicone rubber [J]. Insulating Materials, 2017, 50(12):1-9.

11. Cao Hongwei, Zhu Lina, Wu Wenlong, Du Linjuan, Chen Haojun. A evaluation method for the service life of composite insulators: 201410786920.0 [P].2014-12-18.

12. Zhang Rui. Formula design and aging performance evaluation of silicone rubber materials for composite insulators [J]. Insulators and Surge Arresters, 2012 (6): 39-45.

13. Xuan Sheng, Zhang Younan. Formula of silicone rubber materials for composite insulators in power systems [J]. Synthetic Rubber Industry, 2012, 35(4): 295-290.

14. Zhan Xuegao, Ji Jiaping, Zhuge Yanfei. Development of high-strength thermally vulcanized silicone rubber for composite insulators [J]. Silicone Material, 2010, 24(1): 19-22.

15. Xie Chuan. Study on the status quo of modified asphalt from waste tire rubber powder [J]. China Tire Resources Recycling;CTRA, 02/2018, 23-27.

16. Jiang Xuewen. Analysis of the application prospect, economic and social benefits of rubber asphalt technology [J]. Transportation Construction \& Management, 04/2018, 91-92.

17. Wang Yi, Huang Weidong. Introduction to Rubber Asphalt Use Status in USA [J]. Journal of China \& Foreign Highway, 2009, 29(6):274-277.

18. Cao Rongji, Chen Rongsheng. Experimental study on the influence of rubber asphalt process parameters on its performance [J]. Journal of Southeast University (Natural Science Edition), 2008,38(2):269-273.

19. Li Tinggang, Li Jinzhong, Li Wei. Study on the microscopic mechanism of rubber asphalt and its application in highway engineering [J]. Journal of Highway and Transportation Research and Development, 2011, 28(1): 25-30.

20. Chang Zhanhong. Study on the application of rubber asphalt technology in highway pavement [J]. Transport World, 2018, 468: 52-53.

21. Wang Yonghai. Application of asphalt rubber in the maintenance project of Xueguan Line of Tai'an highway [D]. Jilin University, 12/2017.

22. Wang Xiaofeng, Cao Rongji. Modification mechanism of rubber asphalt [J]. Journal of Chang'an University (Natural Science Edition), 2011, 31(2): 6-11.

23. Wang Shuiyin, Guo Chaoyang, Peng Feng. Modification mechanism of waste tire rubber 
powder [J]. Journal of Chang'an University (Natural Science Edition), 2010, 30(4): 34-38.

24. Yang Ruochong, Tan Zhiming, Huang Xiaoming, et al. Study on road performance of rubber concrete with polymer [J]. China Journal of Highway and Transport, 2010, 23(4); 15-20.

25. Huang S C., Rubber concentrations on rhcology of aged asphalt binders[J]. Journal of Materials in Civil Engineer, 2008, 30(3):221-229.

26. Xiao Chuan, Ling Tianqing. Application and research of waste rubber powder modified asphalt materials in road engineering [J]. Highway Engineering, 2009, 34(4): 49-53.

27. Shen Zhongshuo, Qin Gang, Zhang Zhen, et al. Study on preparation and performance of silicone rubber powder modified asphalt [J]. Highway, 06/2018, 244-248.

28. Writing group of "Rubber Industry". Rubber Industry Handbook, Volume 1 (Revised) [M]. Beijing: Chemical Industry Press, 1989, 538-595.

29. Yang Junhua. Some technological progress of foreign silicone rubber in recent years [J]. China Synthetic Rubber Industry, 1991, 14(5): 372.

30. Dou Qiang, Wu Shishan, Zhu Xuehai, Jin Lin. Study on the blending of methyl vinyl silicone rubber and EPDM [J]. Rubber Industry, 1997, 44(5): 263-266.

31. Zhang Weiying, Li Xiao, Li Jianzhao, Lin Bo. Study on blending of activated modified waste silicone rubber and EPDM [J]. Rubber Industry, 2003, 50(3): 143-146.

32. Sun Bin, Cao Hongwei, Du Linjuan, Du Zhenxia. Study on properties of blends of modified waste silicone rubber composite insulator powder and Ethylene-Propylene-Diene Monomer(EPDM) [J]. Journal of Beijing University of Chemical Technology (Natural Science Edition) , 2017,44(3):33-38.

33. Xie Qianhong, Luo Yongle, Chen Xiaotian, Guo Jiehuang. Research progress of color pavement skidresistant coatings [J]. Guangdong Chemical Industry, 2014,41(14):91-92.

34. Deng Qi, Xu Jinwen, Gao Xinhua, Wu Youli. Technology status quo and development trend of skid-resistant coatings for ship deck [J]. Chinese Journal of Ship Research, 2013, 8(2): 111-116.

35. Zhang Xueqing, Zhang Weiguo, Li Xuchao. Overview of the development of skid-resistant coatings [J]. Modern Paint \& finishing,2002: 5 (3): 10-12. 